DE DE GRUYTER

OPEN

国

BULGARIAN ACADEMY OF SCIENCES

CYBERNETICS AND INFORMATION TECHNOLOGIES • Volume 14, No 2

Sofia $\bullet 2014$

Print ISSN: 1311-9702; Online ISSN: 1314-4081

DOI: $10.2478 /$ cait-2014-0022

\title{
A Combined Method for On-Line Signature Verification
}

\section{Desislava Boyadzhieva, Georgi Gluhchev}

Institute of Information and Communication Technologies, 1113 Sofia

Emails:d.n.dimitrova@gmail.com gluhchev@iinf.bas.bg

\begin{abstract}
A combined method for on-line signature verification is presented in this paper. Moreover, all the necessary steps in developing a signature recognition system are described: signature data pre-processing, feature extraction and selection, verification and system evaluation. NNs are used for verification. The influence of the signature forgery type (random and skilled) over the verification results is investigated as well. The experiments are carried out on SUsig database which consists of genuine and forgery signatures of 89 users. The average accuracy is $98.46 \%$.
\end{abstract}

Keywords: On-line signature verification, neural networks, feature selection, SUsig database, forgery signatures.

\section{Introduction}

Signature verification is the process of confirming the identity, based on the handwritten signature of the user as a form of behavioral biometrics [1]. From one hand, the signatures are a convenient, widely used and secure mean for authentication, and from the other, their input to biometric systems is fast, easy, natural and non-invasive. For these reasons, the problem of signature verification is broadly investigated in the past years. Novel methods and algorithms are developed, mostly for on-line signatures, and lots of them are implemented in practice $[3,8,9]$.

Signature recognition systems are on-line and off-line depending on the signature acquisition method. The off-line method uses a captured image of a written signature after the writing process is over, while the on-line method uses 
devices such as graphical tablets to capture signature during signing and thus a lot of writer's specific features like pressure, speed, pen tilt, azimuth, etc. are available.

In this paper we propose a combined method for signature verification and test it on a SUsig database [2]. For each user of the database we construct a NN model for verification. With regard to this we perform the following steps. At the preprocessing stage, some transformations are performed on the signatures (coordination transformation, rotation and translation). Next, we extract the signature features and perform feature set selection by applying the method for selection of regression variables based on Mallows $C_{p}$ criterion $[5,6]$ to identify the best features subset. We experiment with random and skilled forgeries. After this step, NNs are constructed of varying size of the hidden neurons and a 10-fold crossvalidation is performed in order to choose the best one of them. Such selected model is defined by the following parameters: number of hidden neurons, type of forgery signatures for training and input features. Finally, we train, validate and test all the chosen user's models and obtain the average system accuracy.

This paper is organized as follows. In Section 2 a brief overview over the feature set selection is given. Next, the application of NNs for signature verification is considered in Section 3. The experimental results are presented in Section 4.

\section{Feature set selection}

\subsection{Signature pre-processing}

At first, the signature raw data is acquired by the hardware device (e.g., a graphical tablet, PDA, etc.). To facilitate the feature extraction, it is necessary these raw data to be pre-processed. The operations applied depend on the selected features and the acquisition protocol.

The coordinates $x$ and $y$ of the ink coordinate space are called himetric units $[10]$ and their values fall within $[0,7999] \times[0,5999]$. It is necessary to transform them in the application coordinate system in $[0,1279] \times[0,799]$. This is performed automatically by a method from Microsoft Tablet PC SDK. Since the acquired signatures may be rotated, we have to align them horizontally. The next preprocessing operation is translation of the signatures to a given point of the application coordinate system because it is possible some of the coordinates to obtain negative values after the rotation.

\subsection{Feature extraction}

There are three groups of signature features: global, local and segmental [12]. The global features are extracted for the whole signature, the local features are extracted for each sample point in the signature, and the segmental features are extracted for each signature segment. Over 100 features used in the signature verification are listed in [14].

The extracted global signature features used are presented in Table 1. 
Table 1. Global features

\begin{tabular}{|l|l||l|l|}
\hline $\begin{array}{l}\text { Abbre- } \\
\text { viation }\end{array}$ & Feature name & $\begin{array}{l}\text { Abbre- } \\
\text { viation }\end{array}$ & Feature name \\
\hline A1 & Signature length $L$ & A13 & $\begin{array}{l}\text { Angle of the line between the initial } \\
\text { and end points }\end{array}$ \\
\hline A2 & Signature height $H$ & A14 & $\begin{array}{l}\text { Distance between the leftmost and } \\
\text { center points }\end{array}$ \\
\hline A3 & Height to width ratio $H / L$ & A15 & $\begin{array}{l}\text { Distance between the center and } \\
\text { rightmost points }\end{array}$ \\
\hline A4 & Number of points $N$ & A16 & $\begin{array}{l}\text { Angle of the line between the center } \\
\text { and leftmost points }\end{array}$ \\
\hline A5 & Time duration & A17 & $\begin{array}{l}\text { Angle of the line between the center } \\
\text { and rightmost points }\end{array}$ \\
\hline A6 & Number of segments & A18 & $\begin{array}{l}\text { Distance between the leftmost and } \\
\text { initial points }\end{array}$ \\
\hline A7 & Signature density A4/A1*A2 & A19 & $\begin{array}{l}\text { Distance between the rightmost and } \\
\text { end points }\end{array}$ \\
\hline A8 & $\begin{array}{l}\text { Distance between the initial and } \\
\text { center point }\end{array}$ & A20 & $\begin{array}{l}\text { Angle of the line between the } \\
\text { leftmost and initial points }\end{array}$ \\
\hline A9 & $\begin{array}{l}\text { Distance between the end and center } \\
\text { point }\end{array}$ & A21 & $\begin{array}{l}\text { Angle of the line between the end } \\
\text { and rightmost points }\end{array}$ \\
\hline A10 & $\begin{array}{l}\text { Distance between the initial and end } \\
\text { point }\end{array}$ & A22 & Number of strokes \\
\hline A11 & $\begin{array}{l}\text { Angle of the line between the center } \\
\text { and initial points }\end{array}$ & A23 & Average tilt \\
\hline A12 & $\begin{array}{l}\text { Angle of the line between the center } \\
\text { and end points }\end{array}$ & A24 & Average pressure \\
\hline
\end{tabular}

\subsection{Feature set selection}

Since some features demonstrate higher discriminatory capability than others, feature selection should be performed. This is related to the process of selecting $k$ features of most discrimination power out of $p$ available ones $(k \leq p)$ and it aims to identify and remove as much irrelevant and redundant information, as possible. A review of the processes of feature set selection for signatures is done in [12].

We approach the feature set selection step in signature verification by applying the methods of Hocking, Leslie and LaMotte for selection of regression variables based on Mallows $C_{p}$ criterion for regression [6,7]. This criterion is used to decide on a suitable subset among the contending subsets. It is a measure of the standardized total squared error defined as follows:

$$
C_{p}=\frac{\mathrm{RSS}_{p}}{\widehat{\sigma}^{2}}-(n-2 p) .
$$

In (1) $\mathrm{RSS}_{p}$ denotes the residual sum of squares for the particular regression with $p$ variables and $\hat{\sigma}^{2}$ is an estimate of the residual mean square $\sigma^{2}$ for full regression.

By applying the methods of Hocking, Leslie and LaMotte we identify the best feature subsets of various size for each user on the basis of his/her eight or ten genuine signatures and ten random forgeries. Among these subsets we select the best subset that has a $C_{p}$ value closest to $p$, where $p$ is the number of regression coefficients. Thus, for each user we obtain the best feature subset of different size. An extensive overview of this method may be found in [4]. 


\section{Neural networks for signature verification}

Neural networks are suitable to be used for signature verification since they are an excellent generalization tool (under normal conditions) and are a useful mean of coping with the diversity and variations inherent in handwritten signatures [13]. Usually, a particular NN is built for each user on the basis of his/her genuine and forgery signatures. The number of the input neurons is $p$ where $p$ is the number of the features. The single output neuron has a value 1 for the genuine signature and a value 0 for the forgery signature. After training, a score threshold is determined. If the verification result (at the time of testing a signature) is greater than the corresponding score threshold, the signature is considered genuine, otherwise forgery. This approach is widespread because it allows fast adding and deleting of signatures for new and existing users [13]. Usually, NN training takes much time but in this approach it is done off-line, so that the users are not forced to wait.

\section{Experiments}

The experiments are carried out in MATLAB environment. We use Neural Network Toolbox.

\subsection{Signature database SUsig}

The signature database used in the experiments is SUsig [2]. It consists of two sub corpuses: Visual and Blind. We test the proposed combined method on signatures of Blind subcorpus. There are 89 users and for each of them 8 or 10 genuine and 10 skilled forgeries are acquired by using a graphical tablet Wacom Graphire2. The genuine signatures are collected in a single session. The signature data consists of the following information for each of the signature points: $x$ and $y$ coordinates, timestamp, pressure level and a pen up or down indicator.

\subsection{Results}

We experiment with:

(1) A common feature set for all users

At first, we identify the significant correlation coefficients pairs at 0.01 confidence level, 99\% confidence interval for all the users and then build the corresponding histogram. After that, we find the feature pairs which are met in more than $25 \%$ of the users and remove one feature out of a pair by applying the method of correlation pleiads [7]. In this way, the feature number is reduced by around $50 \%$ and the remaining features are $\mathrm{A} 1, \mathrm{~A} 2, \mathrm{~A} 4, \mathrm{~A} 6, \mathrm{~A} 10, \mathrm{~A} 12, \mathrm{~A} 13, \mathrm{~A} 16$, A17, A21, A22, A23, A24.

(2) An individual feature subset

At first, we identify the significant correlation coefficients at 0.01 confidence level for each user. Then we remove one feature out of a pair by applying the method of correlation pleiads [7]. Next, we apply the method of Hocking, Leslie and LaMotte [5] to the remaining features. Let us denote by Variant 1 the case, in 
which the feature subset is determined by using the genuine and random forgery signatures and denote by Variant 2 the case, in which the feature subset is determined by using the genuine and skilled forgery signatures. The size of the obtained $p$-subset and the corresponding number of users are specified in Table 2.

Table 2. Individual $p$-subsets
\begin{tabular}{|c|c||c|c|}
\hline \multicolumn{2}{|c||}{ Variant 1} & \multicolumn{2}{c|}{ Variant 2} \\
\hline Size of $p$-subset & Number of users & Size of $p$-subset & Number of users \\
\hline 9 & 42 & 9 & 48 \\
\hline 8 & 5 & 8 & 9 \\
\hline 7 & 7 & 7 & 4 \\
\hline 6 & 12 & 6 & 10 \\
\hline 5 & 14 & 5 & 15 \\
\hline 4 & 5 & 4 & 3 \\
\hline 3 & 4 & 3 & 0 \\
\hline
\end{tabular}

There is a significant feature reduction for both Variant 1 and Variant 2, since their initial number (13) is reduced down to 9 for about half of the users, reduced down to 5 or 6 features for $30 \%$ of the users.

In Table 3 all the six NN models are described together with their parameters. Let us denote by Case 1 the case, in which only random forgeries are used for NN training, and denote by Case 2 the case, in which both random and skilled forgeries are used.

Table 3. Parameters of the NN models

\begin{tabular}{|c|c|c|c|c|c|}
\hline $\begin{array}{l}\text { \# of } \\
\text { model }\end{array}$ & $\begin{array}{c}\text { Features } \\
\text { (input neurons) }\end{array}$ & $\begin{array}{l}\text { Genuine } \\
\text { signatures }\end{array}$ & \multicolumn{2}{|c|}{$\begin{array}{l}\text { Forgery } \\
\text { signatures }\end{array}$} & $\begin{array}{c}\text { Number of hidden } \\
\text { neurons }\end{array}$ \\
\hline 1 & Common set & \multirow{6}{*}{8 or 10} & \multirow{3}{*}{$\begin{array}{c}15 \\
\text { random }\end{array}$} & \multirow{3}{*}{ Case 1} & \multirow{6}{*}{ From 1 up to 5} \\
\hline 2 & Variant 1 & & & & \\
\hline 3 & Variant 2 & & & & \\
\hline 4 & Variant 2 & & \multirow{3}{*}{$\begin{array}{l}9 \text { random and } \\
6 \text { skilled }\end{array}$} & \multirow{3}{*}{ Case 2} & \\
\hline 5 & Variant 1 & & & & \\
\hline 6 & Common set & & & & \\
\hline
\end{tabular}

All 30 models are evaluated by a 10-fold cross validation for each user and the best performed optimal NN model is selected together with its parameters: number of hidden neurons, type of signature forgeries for training and input features.

In Table 4 the number of users for all the chosen models is given. Model \# 2 ( $30 \%$ of the users) is the most common, followed by model \# 4 (18\% of the users). The number of the models trained on individual features (Variant 1 ) is $53(60 \%$ of the users).

Table 4. Number of model occurrences

\begin{tabular}{|l|c|c|c|}
\hline \multicolumn{1}{|c|}{ User } & Case & Model \# & Number of users \\
\hline Common features & Case 1 & 1 & 13 \\
\hline Variant 1 & Case 1 & 2 & 27 \\
\hline Variant 2 & Case 1 & 3 & 13 \\
\hline Common features & Case 2 & 4 & 16 \\
\hline Variant 1 & Case 2 & 5 & 9 \\
\hline Variant 2 & Case 2 & 6 & 11 \\
\hline
\end{tabular}




\subsection{Results interpretation}

In the current scenario: (1) it is demonstrated that each user has its own discriminative feature subset. In other words, we cannot restrict to a common feature set valid for all users, but instead of that we have to consider each user best feature subset separately; (2) The initial feature set size is reduced to a higher extent if random forgeries (Var. 1) are used for building the regression model for Hocking, Leslie and LaMotte method instead of skilled forgeries; (3) It cannot be concluded that the models built only on random forgery signatures perform better than those built on both random and skilled forgeries; (4) The obtained results are satisfactory, demonstrating verification accuracy of $98.46 \%$, EER $1.61 \%$, FRR $0 \%$, FAR $2.70 \%$. These results are similar to those obtained on the same SUsig database by applying a linear classifier [11].

\section{References}

1. N a 1 w a, V. S., I. E k e 1 a n d. Automatic On-Line Signature Verification. - In: Proceedings of the IEEE'85, 1997, 213-239.

2. Kholmatov, A., B. Yanikoglu. SUSIG: An On-Line Signature Database, Associated Protocols and Benchmark Results. - Pattern Analysis \& Applications, Vol. 12, 2009, 227-236.

3. J a i n, A., L i S t a n. Encyclopedia of Biometrics. Springer, 2009.

4. Boyadzhieva, D., G. Gluhchev. Feature Set Selection for On-Line Signatures Using Selection of Regression Variables. - In: Proceedings of 4th International Conference on Pattern Recognition and Machine Intelligence PReMI'11, 2011, 440-445.

5. Hocking, R. R., R. Leslie. Selection of the Best Subset in Regression Analysis. Technometrics, Vol. 9, 1967, 531-540.

6. L a M o t t e, L. R., R. R. Hock ing. Computational Efficiency in the Selection of Regression Variables. - Technometrics, Vol. 12, 1970, 83-93.

7. A y v a z y a n, S. A., Z. I. B e j a e va, O. V. S t a roverov. Classification of multidimensional observations. Moscow, Statistics, 1974, p. 240 (in Russian).

8. Gluhchev, G., M. Savov, O. Boumbarov, D. Vassileva. A New Approach to Signature Based Authentication. - In: 2nd Int. Conf. on Biometrics, Seoul, 26-29 August, 2007, 594-603.

9. S a v o v, M., G. Glu h c h e v. Signature Verification Via Hand-Pen Motion Investigation. - In: 10. Ink Data. Proc. Int. Conf. "Recent Advances in Soft Computing", Canterbury, 2006, 490-495. http://msdn.microsoft.com/en-us/library/ms811395.aspx

11. Kholmatov, A., B. Yanikoglu. Identity Authentication Using Improved On-Line Signature Verification Method. - Pattern Recognition Letters, Vol. 26, 2005, No 15, 2400-2408.

12. Richiardi, J., H. Ketabdar, A. Drygajl o. Local and Global Feature Selection for OnLine Signature Verification. - In: Eighth International Conference on Document Analysis and Recognition (ICDAR'05), 2005, 625-629.

13. $\mathrm{McCabe}$, A., J. Trevathan, W. Re a d. Neural Network-Based Handwritten Signature Verification. - Journal of Computers, Vol. 3, 2008, No 8, 9-22.

14. L e c l e r c, F., R. P l a m ond on. Automatic Signature Verification: The State of the Art 19891993. - International Journal of Pattern Recognition and Artificial Intelligence, Vol. 8, 1994, No 3, 643-660. 